\title{
Sivas İli Heterocera (Lepidoptera) Faunasına Katkılar
}

\author{
Erdem SEVEN ${ }^{1}$ \\ Kesran $\mathrm{AKIN}^{2}$ \\ ${ }^{1}$ Batman Üniversitesi, Turizm İşletmeciliği ve Otelcilik YO, Batman \\ ${ }^{2}$ Bitlis Eren Üniversitesi, Fen Edebiyat Fakültesi, Biyoloji Bölümü, Bitlis \\ $\triangle$ : erdem_seven@hotmail.com
}

\begin{abstract}
ÖZET: Bu çalışmada, Sivas ilinden Lepidoptera takımına bağlı 11 familyaya: Cosmopterigidae (1), Crambidae (13), Ethmiidae (1), Gelechiidae (2), Geometridae (6), Hepialidae (1), Lymantriidae (1), Lasiocampidae (1), Noctuidae (13), Pyralidae (11) ve Tortricidae (3) ait 53 tür sunulmuştur. Bu türlerin tamamı araştırma alanı için yeni kayıttır. Ayrıca, bunlar arasında yer alan bazı nadir türlerin Türkiye'deki yayılışları tartışılmıştır.
\end{abstract}

Anahtar Kelimeler: Lepidoptera, Heterocera, fauna, Sivas, Türkiye

\section{Contributions to the Knowledge of Heterocera (Lepidoptera) Fauna of Sivas Province}

ABSTRACT: In this study, in Lepidoptera order, 53 species belonging to 11 families including Cosmopterigidae (1), Crambidae (13), Ethmiidae (1), Gelechiidae (2), Geometridae (6), Hepialidae (1), Lymantriidae (1), Lasiocampidae (1), Noctuidae (13), Pyralidae (11) and Tortricidae (3) are presented from Sivas province. All of these species are new records for the research area. In addition, the distribution of some rare species in Turkey are discussed.

Key Words: Lepidoptera, Heterocera, fauna, Sivas, Turkey

\section{GíRiș}

Türkiye Lepidoptera faunasıyla ilgili eserler çoğunlukla taksonomik, faunistik ve zoocoğrafik araştırmalara dayanmaktadır. Çok eski araştırmalar daha çok yabancı entomologlar tarafından genellikle Anadolu'dan toplanan örnekler üzerinedir. 20. yüzyılın sonlarına doğru yerli araştırmacıların da çalışmalarının artması ve katkıları, ülkemiz Lepidoptera faunasıyla ilgili daha net sonuçların ortaya konulmasını sağlamıştır. Türkiye'deki Lepidoptera türü sayısı, en son yayınlanan araştırma verilerine göre, 5363 tür (Koçak, 2014) olarak sunulmaktadır.

Sivas ilinin, güve faunasıyla ilgili çok kaynak bulunmamakla birlikte, Heterocera türleri için doğrudan veya dolaylı olarak önemli çalışmalar; Zukowsky (1937, 1938, 1941), Lattin (1950, 1951), Hacker (1985, 1986, 1987a, 1987b), Hacker ve ark. (1987), Hacker ve Lödl (1989), Seven (1996), Koçak ve Seven (1996), Okyar ve Ataç (1999), Koçak ve Kemal (2009), Kemal ve Koçak (2015) ve Özdemir'in (2016) çalışmalarıdır. Sivas Lepidoptera faunasının tespitine yönelik son güncel liste, Kemal ve Koçak (2015) tarafindan yayınlanmıştır. Bu listeye göre 684 güve türünün Sivas'ta yayılış gösterdiği belirtilmiştir.

$\mathrm{Bu}$ çalışmanın amacı, Sivas ili Heterocera (Lepidoptera) faunasına katkıda bulunmaktır. Araştırma sonucunda Sivas ili Heterocera faunasına, 11 familyaya ait [Cosmopterigidae (1), Crambidae (13), Ethmiidae (1), Gelechiidae (2), Geometridae (6), Hepialidae (1), Lymantriidae (1), Lasiocampidae (1), Noctuidae (13), Pyralidae (11) ve Tortricidae (3)] toplam 53 tür yeni kayıt olarak ilave edilmiştir. Önemli doğa alanlarını içeren Sivas ilinde (Eken ve ark., 2006), ileride yapılacak kapsamlı araștırmalarla, Lepidoptera tür sayısının daha da artacağı düşünülmektedir.

\section{MATERYAL VE METOT}

Örnekler, 2015 yılında Sivas ilindeki iki farklı lokaliteden (Çizelge 1.), 1şık tuzağıyla (Ultraviyole led) yakalanmıştır. Toplanan örnekler, iğnelenip etiketlenerek naftalinli kutulara yerleştirilmiştir. Daha sonra yumuşatılarak gerilen materyallerin, öncelikli olarak dış morfolojik karakterleri, yetersiz kaldığı durumlarda ise genital yapıları da dikkate alınarak teşhisleri yapılmıştır. Genital preparatlar hazırlanırken, genel hatlarıla Robinson'un (1976) prosedürü takip edilmiştir. Türlerin teşhis işlemlerinde, Bleszynski (1965), Sattler (1967), Razowski (1970), Roesler (1973, 1993), Fazekas (1991), Fibiger (1997), Parenti (2000), Hausmann (2001, 2004), Hacker (2004), Goater ve ark. (2005), Slamka (2006 (2011), 2008, 2013), Hausmann ve Viidalepp (2012), Leraut (2014) ve Karsholt ve Sumpich'ın (2015) çalışmalardan faydalanılmıştır. Sistematik tayini yapılan türler, Batman Üniversitesi, Fen Edebiyat Fakültesi, Biyoloji Laboratuvarı ile Bitlis Eren Üniversitesi, Fen Edebiyat Fakültesi, Biyoloji Laboratuvarında muhafaza edilerek saklanmaktadır.

Teşhis işlemleri sonucunda, alandan Heterocera grubuna ait 111 tür tespit edilmiştir. Bu türlerden sadece Sivas ili için yeni kayıt özelliği olan 53 tür bu çalışmada listelenmiştir. Türler bulgular kısmında alfabetik sıraya göre; familya, tür adı, sinonimler (Koçak ve Kemal, 2009; Koçak, 2014), lokalite (Çizelge 1'de belirtilen kodlarla) ve çalışılan materyal kategorileri şeklinde düzenlenerek sunulmuştur. 
Çizelge 1. Örneklerin toplandığı lokalite bilgileri

\begin{tabular}{llllll}
\hline Kodu & Lokalite & Yükseklik & Tarih & Koordinatlar & Habitat tipi \\
\hline Cuk & Cumhuriyet Üniversitesi & $1280 \mathrm{~m}$ & $02-04.09 .2015$ & $39^{\circ} 41^{\prime} \mathrm{K}-37^{\circ} 01^{\prime} \mathrm{D}$ & Seyrek çam ormanı \\
& Kampüsü & & & & \\
Ml & Mescidli & $1250 \mathrm{~m}$ & 08.09 .2015 & $39^{\circ} 38^{\prime} \mathrm{K}-37^{\circ} 00^{\prime} \mathrm{D}$ & Dağlık step alan \\
\hline
\end{tabular}

\section{BULGULAR}

Cosmopterigidae

Pyroderces argyrogrammos (Zeller, 1847)

Synonym(s): argyrogrammos Zeller, goldeggiella Herrich-Schäffer, [1853]

Lokalite: Ml, Materyal: 2 우

Crambidae

Agriphila bleszynskiella Amsel, 1961

Synonym(s): bleszynskiella Amsel, 1961

Lokalite: M1, Materyal: 1

Agriphila inquinatella ([Denis \& Schiffermüller], 1775)

Synonym(s): inquinatella [Denis \& Schiffermüller], 1775; ? arbustella Schrank, 1802; nevadensis Caradja, 1910

Lokalite: Cuk, Materyal: $1 \hat{\jmath}$

Agriphila paleatella (Zeller, 1847)

Synonym(s): paleatellus Zeller, 1847

Lokalite: Ml ve Cuk, Materyal: 7 § 3 ㅇ

Agriphila tersella (Lederer, 1855)

Synonym(s): tersellus Lederer,1855; graphellus Constant, 1884; hungaricus Schmidt, 1909; austellus Chrétien, 1913; uniformellus Caradja, 1918; chneouri Lucas, 1942

Lokalite: Ml ve Cuk, Materyal: 1 ô 1 ㅇ

Agriphila tristella ([Denis \& Schiffermüller], 1775)

Synonym(s): tristella [Denis \& Schiffermüller], 1775; ferruginella Thunberg, 1788; paleella Hübner, 1796; aquilella Hübner, 1796; moerens Fabricius, 1798; fuscinella Schrank, 1802; fuscelinellus Stephens, 1834; ribbeellus Caradja, 1910; discistrigatus Hampson, 1919

Lokalite: Ml ve Cuk, Materyal: 5 ๙ 8 ㅇ

Catoptria confusella (Staudinger, 1881)

Synonym(s): confusellus Staudinger, 1881

Lokalite: Ml, Materyal: 1 $\delta$

Catoptria dimorphella (Staudinger, 1881)

Synonym(s): dimorphellus Staudinger, 1881; haywardi Rebel, 1939; wolfi Ganev \& Hacker, 1984

Lokalite: Ml, Materyal: $2 \lesssim 1$ q

Cynaeda dentalis ([Denis \& Schiffermüller], 1775)

Synonym(s): dentalis [Denis \& Schiffermüller], 1775; ramalis Fabricius, 1787; radiata Esper, 1788; fulminans Fabricius, 1794; dilutalis De Lattin, 1959

Lokalite: Ml, Materyal: 20 1775)

Dolicharthria punctalis ([Denis \& Schiffermüller], 1775

Synonym(s): punctalis [Denis \& Schiffermüller],

Lokalite: Ml, Materyal: 5 ㅇ

Ecpyrrhorrhoe diffusalis (Guenée, 1854)

Synonym(s): diffusalis Guenée, 1854; tenuialis Mann, 1862

Lokalite: Ml, Materyal: 2 ठ

Euchromius bellus (Hübner, 1796)
Synonym(s): bella Hübner, 1796; bellalis Hübner, [1825]

Lokalite: M1, Materyal: 1 \%

Evergestis nomadalis Lederer, 1871

Synonym(s): nomadalis Lederer, 1871

Lokalite: Ml, Materyal: 1 ऽ

Paracorsia repandalis ([Denis \& Schiffermüller], 1775)

Synonym(s): repandalis [Denis \& Schiffermüller], 1775

Lokalite: M1, Materyal: $1 \curvearrowright$

Ethmiidae

Ethmia bipunctella (Fabricius, 1775)

Synonym(s): bipunctella Fabricius, 1775; echiella [Denis \& Schiffermüller], 1775; hochenwartiella Rossi, 1790; griseicostella Wiltshire, 1947

Lokalite: M1, Materyal: 1 त

Gelechiidae

Nothris sabulosella Rebel, 1935

Synonym(s): sabulosella Rebel, 1935

Lokalite: Ml, Materyal: 1 ठै

Nothris verbascella (Brahm, 1791)

Synonym(s): \#verbascella [Denis \& Schiffermüller], 1775; verbascella Brahm, 1791; lutarea Haworth, 1828; clarella Amsel, 1935

Lokalite: M1, Materyal: 1 \%

Geometridae

Casilda antophilaria (Hübner, [1813])

Synonym(s): antophilaria Hübner, [1813]; gegenaria Alpheraky, 1883; \#albipunctaria Alpheraky, 1883; elvira Thierry-Mieg, 1911

Lokalite: Cuk, Materyal: 1 ๙

Dyscia (Calodyscia) innocentaria (Christoph, 1885)

Synonym(s): innocentaria Christoph, 1885; sicanaria Oberthür, 1923

Lokalite: Ml ve Cuk, Materyal: 5 §ิ 3 q

Charissa (Kemtrognophos) zeitunaria (Staudinger, 1901)

Synonym(s): zeitunaria Staudinger, 1901

Lokalite: Ml, Materyal: 1 ઈิ 1 ㅇ

Peribatodes rhomboidarius [Denis \&

Schiffermüller], 1775)

Synonym(s): \#rhomboidaria [Denis \& Schiffermüller], 1775; inaequalis Goeze, 1781; gemmaria Brahm, 1791; ichnusaria Ghiliani, 1852; corsicaria Schawerda, 1931; saerdabensis Wehrli, 1943

Lokalite: Ml ve Cuk, Materyal: 1 ภ 2 ㅇ

Phaiogramma etruscaria (Zeller, 1849)

Synonym(s): etruscaria Zeller, 1849; pulmentaria

Guenée, [1858]; palaestinensis Fuchs, 1903

Lokalite: Cuk, Materyal: 1 đ

Scopula turbulentaria (Staudinger, 1870) 
Synonym(s): turbulentaria Staudinger, 1870; collata W.Warren, 1901; habenata W.Warren, 1901; syriacata Neuburger, 1904; syriturcica Wehrli, 1934

Lokalite: M1, Materyal: $2 \sigma^{\lambda}$

Lasiocampidae

Lasiocampa grandis (Rogenhofer, 1891)

Synonym(s): grandis Rogenhofer, 1891

Lokalite: Cuk, Materyal: 3 ऽ 1

Hepialidae

Triodia amasina (Herrich-Schäffer, [1851])

Synonym(s): amasina Herrich-Schäffer, [1851]

Lokalite: Cuk, Materyal: 4 ○े 3

Lymantriidae

Parocneria signatoria (Christoph, 1893)

Synonym(s): signatoria Christoph, 1893

Lokalite: Ml, Materyal: $2 \delta^{\lambda}$

Pyralidae

Ancylosis (s.str.) cinnamomella (Duponchel, 1836)

Synonym(s): dilutella Tr., 1832 [n.o.]; cinnamomella

Dup., 1836; labeomella Staudinger, 1859; lafauryella

Rag., 1887; byzacaeniella Rag., 1887; roseopennella

Rag., 1901; tunesella Caradja, 1910; fuliginosa Rothschild, 1913; pictella Tur., 1922; ferrugineolineella Tur., 1924

Lokalite: Ml, Materyal: 1 ㅇ

Pempelia albariella Zeller, 1839

Synonym(s): albariella Zeller, 1839

Lokalite: Ml, Materyal: 1

1775)

Pempelia palumbella ([Denis \& Schiffermüller],

Synonym(s): palumbella [Denis \& Schiffermüller], $1775(\mathrm{Ml})$

Lokalite: Ml ve Cuk, Materyal: 1 ㅇ

Phycita strigata (Staudinger, 1879)

Synonym(s): strigata Staudinger, 1879

Lokalite: Ml, Materyal: 1 o 3 ㅇ

Phycitodes albatella (Ragonot, 1887)

Synonym(s): albatella Ragonot, 1887

Lokalite: Ml ve Cuk, Materyal: $5 \lesssim 7$ ำ

Phycitodes lacteella (Rothschild, 1915)

Synonym(s): lacteella Rothschild, 1915; viettella Roesler, 1965

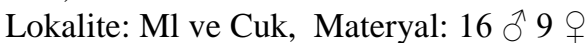

Phycitodes nigrilimbella (Ragonot, 1887)

Synonym(s): nigrilimbella Ragonot, 1887

Lokalite: Ml ve Cuk, Materyal: 3

Psorosa dahliella (Treitschke, 1832)

Synonym(s): dahliella Treitschke, 1832; bivitella

Duponchel, 1836

Lokalite: Ml, Materyal: $1 \lesssim$

Pyralis perversalis Herrich-Schäffer, 1849

Synonym(s): perversalis Herrich-Schäffer, 1849; transcaspica Rebel, 1903; \#transcaspia Speidel, 1996

Lokalite: Ml, Materyal: 1 ô 1 ㅇ

Selagia spadicella (Hübner, 1796)

Synonym(s): spadicella Hübner, 1796

Lokalite: Cuk, Materyal: 3 त 1 q

Stemmatophora brunnealis (Treitschke, 1829)

Synonym(s): brunnealis Treitschke, 1829; phaealis

Hampson, 1900
Lokalite: Ml ve Cuk, Materyal: 6 ภ 2 ㅇ

Noctuidae 1775)

Epilecta linogrisea ([Denis \& Schiffermüller],

Synonym(s): linogrisea [Denis \& Schiffermüller], 1775; lutosa Staudinger, 1901

Lokalite: Ml, Materyal: 2 ๙ 1 ㅇ 1794)

Eublemma (candidana-gr.) minutata (Fabricius,

Synonym(s): minutata Fabricius, 1794; noctualis Hübner, 1796; paula Hübner, [1809]

Lokalite: M1, Materyal: $2 \widehat{\sigma}$

Euxoa (s.str.) hastifera (Donzel, 1847)

Synonym(s): hastifera Donzel, 1837; proleuca Hampson, 1903; pomazensis Kovacs, 1952; hungarica Kovacs, 1952

Lokalite: M1, Materyal: 2 ठ

Euxoa (s.str.) glabella (Wagner, 1930)

Synonym(s): glabella Wagner, 1930

Lokalite: M1, Materyal: 2 ๙ 1

Euxoa (s.str.) aquilina ([Denis \& Schiffermüller], 1775)

Synonym(s): aquilina [Denis \& Schiffermüller], 1775; fictilis Hübner, [1813]; unicolor Hübner, [1813]; petrina Mayer, 1937

Lokalite: Ml, Materyal: 2 ภ 2 q

Caradrina (Paradrina) flavirena (Guenée, 1852)

Synonym(s): flavirena Guenée, 1852; minor Staudinger, 1897; subdita W.Warren, 1911; muricolor Boursin, 1933; subdita Leraut, 1980

Lokalite: Ml ve Cuk, Materyal: $6 \curvearrowright 1$ 우

Chersotis (s.str.) rectangula (Fabricius, 1787)

Synonym(s): rectangula Fabricius, 1787

Lokalite: Ml, Materyal: 3 ठे 1775)

Hoplodrina blanda ([Denis \& Schiffermüller],

Synonym(s): blanda [Denis \& Schiffermüller], 1775; redacta Haworth, 1809; taraxaci Hübner, [1813]

Lokalite: M1, Materyal: 2 ठ 1 ㅇ

Luperina rjabovi (Kljutschko,1967)

Synonym(s): rjabovi Kljutschko, 1967

Lokalite: Ml ve Cuk, Materyal: 3 ก 3 o

Mythimna (Hyphilare) albipuncta (Fabricius, 1787)

Synonym(s): \#albipuncta [Denis \& Schiffermüller], 1775; albipuncta (Fabricius, 1787); rufotincta Wagner, 1921

Lokalite: M1, Materyal: 7 ๙ 4 ㅇ 1775)

Mesoligia furuncula ([Denis \& Schiffermüller],

Synonym(s): furuncula [Denis \& Schiffermüller], 1775; spoliatricula [Denis \& Schiffermüller], 1775; bicoloria Villers, 1789; vinctuncula Hübner, [1803]; humeralis Haworth, 1809; terminalis Haworth, 1809; rufuncula Haworth, 1809

Lokalite: Cuk, Materyal: 3

Xestia (s.str.) sareptana (Herrich-Schäffer, [1851])

Synonym(s): sareptana Herrich-Schäffer, [1851]; iobaphes Boursin, 1936

Lokalite: Ml, Materyal: 1 §ิ 1 ㅇ 


\section{Xestia (s.str.) xanthographa ([Denis \& Schiffermüller], 1775) \\ Synonym(s): $\quad$ xanthographa \\ [Denis \&}

Schiffermüller], 1775; tetragona Haworth, 1809;

budensis Freyer, 1838; elutior Alpheraky, 1887; lepida Constantinio, 1922

Lokalite: Ml, Materyal: $2 \lesssim 1$ ㅇ

Tortricidae

Aethes dilucidana (Stephens, 1852)

Synonym(s): dilucidana Stephens, 1852

Lokalite: Ml, Materyal: 20

Cochylis posterana Zeller, 1847

Synonym(s): posterana Zeller, 1847; nubivagana Zerny, 1935

Lokalite: Cuk, Materyal: 2

Phalonidia contractana (Zeller,1847)

Synonym(s): contractana Zeller, 1847; manifestana Kennel, 1900; exaequata Meyrick, 1923; thermoconis Meyrick, 1925

Lokalite: Ml, Materyal: 4 ${ }^{\Uparrow} 2$ ㅇ

\section{TARTIŞMA ve SONUÇ}

Sivas ilinden 684 güve türü bilinmekte olup, bunlardan Heterocera içerisindeki Cosmopterigidae, Crambidae, Gelechiidae, Geometridae, Lymantriidae, Lasiocampidae, Noctuidae, Pyralidae ve Tortricidae familyalarına ait ise 561 tür bilinmektedir (Kemal ve Koçak, 2015). Bu çalı̧̧mada, Çizelge 2.'de belirtilen familyalara toplam 53 türün ilavesiyle bu sayı 614'e, ildeki toplam güve sayısı ise 737'ye yükselmiştir. Bunlar arasindan en fazla ilave tür sayısı Crambidae ve Noctuidae familyalarından olmuştur. Ayrıca Ethmiidae (Ethmia bipunctella (Fabricius, 1775) ve Hepialidae (Triodia amasina (Herrich-Schäffer, [1851])) familyaları alanının faunasına yeni kayıt olarak verilmiştir (Çizelge 2).

Çizelge 2. Familyalara göre Sivas'tan bilinen ve bu çalışmayla Sivas'a ilave olan Heterocera tür sayıları

\begin{tabular}{lccc} 
Familya & $\begin{array}{c}\text { Bilinen } \\
\text { tür say1s1 }\end{array}$ & $\begin{array}{c}\text { İlave tür } \\
\text { sayıs1 }\end{array}$ & Toplam \\
\hline Cosmopterigidae & 4 & 1 & 5 \\
Crambidae & 19 & 13 & 32 \\
Ethmiidae & 0 & 1 & 1 \\
Gelechiidae & 3 & 2 & 5 \\
Geometridae & 75 & 6 & 81 \\
Hepialidae & 0 & 1 & 1 \\
Lymantriidae & 3 & 1 & 4 \\
Lasiocampidae & 8 & 1 & 9 \\
Noctuidae & 397 & 13 & 410 \\
Pyralidae & 46 & 11 & 57 \\
Tortricidae & 6 & 3 & 9 \\
Toplam & $\mathbf{5 6 1}$ & $\mathbf{5 3}$ & $\mathbf{6 1 4}$ \\
\hline
\end{tabular}

Araştırma sonucuna göre Türkiye'deki yayılışları bakımından nadir olan, Kars (Koçak ve Kemal, 2009) ve Siirt'ten (Seven, 2014) bilinen Parocneria signatoria (Christoph, 1893) (Lymantriidae); Malatya (Koçak ve Kemal, 2009) ve Bitlis (Akın, 2014) illerinden bilinen Agriphila bleszynskiella Amsel, 1961 (Crambidae); ülkemizde sadece Konya'dan (Koçak ve Kemal, 2009) bilinen Nothris sabulosella Rebel, 1935 (Gelechiidae); Amasya, Kahramanmaraş (Koçak ve Kemal, 2009) ve Bitlis'ten (Akın, 2014) bilinen Stemmatophora brunnealis (Treitschke, 1829); Erzurum, Kahramanmaraş (Koçak ve Kemal, 2009) ve Bitlis (Akın, 2014) illerinden bilinen Psorosa dahliella (Treitschke, 1832) (Pyralidae); Burdur ve Isparta'dan (Koçak ve Kemal, 2009) bilinen Aethes dilucidana (Stephens, 1852) (Tortricidae) türleri Sivas ilinden de tespit edilerek, bu türlerin yayılış alanları genişletilmiştir.

\section{KAYNAKLAR}

Akın K 2014. Mutki ilçesi (Bitlis) Pyralidae (Lepidoptera) faunası ve ekolojisi üzerine araştırmalar. YYÜ. Fen Bil. Enst., Biyoloji ABD, Doktora Tezi. [Yayınlanmamıs]. xxii $+340 \mathrm{~s}$.

Bleszynski S 1965. Crambinae, [In] Amsel, H. G., Reisser, H. \& F. Gregor: Microlepidoptera Palaearctica vol. 1. Georg Fromme \& Co.,Wien, 133 pls. xlvii $+533 \mathrm{pp}$.

Eken G, Bozdoğan M, İsfendiyaroğlu S, Kılıç DT, Lise Y 2006. Türkiye'nin Önemli Doğa Alanları. Doğa Derneği. Ankara, Cilt(2): 1-639.

Fazekas I 1991. Phtheochroa Annae Huemer, 1990 Und Agriphila Beioniella Zerny, 1914 Als Neue Arten Im Bakony-Gebirge (Ungarn) Lepidoptera: Tortricidae et Pyralidae. Folia Musei Historico-naturalis Bakonyiensis, 10: 59-66.

Fibiger M 1997. Noctuidae Europaeae. Vol. 3, Noctuinae. Entomological Press, 1-418.

Goater B, Nuss M, Speidel W 2005. Pyraloidea I (Crambidae: Acentropinae, Evergestinae, Heliothelinae, Schoenobiinae, Scopariinae). - In P. Humer \& O. Karsholt (eds.): Microlepidoptera of Europe 4, 1-304.

Hacker H 1985. Dritter Beitrag zur Erfassung der Noctuidae der Türkei. Beschreibung neuer Taxa, Erkenntnisse zur Systematik der kleinasiatischen Arten und faunistisch bemerkenswerte Funde aus den Aufsammlungen von Hacker und Wolf aus dem Jahr 1984 (Lepidoptera). Neue Ent. Nachr., 15: 1-65.

Hacker H 1986. 2. Beitrag zur Erfassung der Noctuidae der Türkei. Beschreibung neuer Taxa, Erkenntnisse zur Systematik der kleinasiatischen Arten und faunistisch bemerkenswerte Funde aus den Aufsammlungen von de Freina aus den Jahren 19761983 (Lepidoptera). Spixiana 9 (1): 25-81, figs Hacker,H., 1990, Die Noctuidae Vorderasiens (Lepidoptera). Neue ent. Nachr., 27: 1-707.

Hacker H 1987a. Erster Beitrag zur systematischen Erfassung der Noctuidae der Türkei (Lepidoptera). Atalanta, 17(1/4) (1986): 1-26.

Hacker H 1987b. Fünfter Beitrag zur systematischen Erfassung der Noctuidae der Türkei. Beschreibung neuer Taxa und faunistisch bemerkenswerte Funde aus der Aufsammlungen von Wolf und Hacker aus dem Jahr 1985 sowie Ergänzungen zu früheren Arbeiten (Lepidoptera). Atalanta, 17(1/4) (1986): 2783. 
Hacker H 2004. Revision of the genus Caradrina Ochsenheimer, 1816 with notes on other genera of the tribus Caradrini (Lepidoptera, Noctuidae). Esperiana, 10: 7-690.

Hacker H, Kuhna P, Gross FJ 1987. 4. Beitrag zur Erfassung der Noctuidae der Türkei. Beschreibung neuer Taxa, Erkenntnisse zur Systematik der kleinasiatischen Arten und faunistisch bemerkenswerte Funde aus den Aufssamlungen von Gross und Kuhna aus den Jahren 1968-1984 (Lep., Noctuidae) Synonym(s): Mitt. münch. ent. Ges., 76(31.12.1986): 79-141.

Hacker H, Lödl M 1989. Taxonomisch und faunistisch bemerkenswerte Funde aus der Sammlung Pinker im Naturhistorischen Museum Wien. Neunter Beitrag zur systematischen Erfassung der Noctuidae (Lepidoptera) der Türkei. Z. ArbGem. Öst. Ent., 40(1/2): 65-82.

Hausmann A 2001. The Geometrid Moths of Europe Vol. I. Apollo Books, Stenstrup, Denmark, 282 pp.

Hausmann A 2004. The Geometrid Moths of Europe: Sterrhinae, Vol. 2. Apollo Books, Denmark, 600 pp.

Hausmann A, Viidalepp J 2012. Larentiinae I. In: Hausmann, A, editor. The Geometrid Moths of Europe 3. Apollo Books, Stenstrup, 743 pp.

Karsholt O, Sumpich J 2015. A review of the genus Nothris Hübner, 1825, with description of new species (Lepidoptera: Gelechiidae). Zootaxa, 4059(3): 471-498.

Kemal M, Koçak AÖ 2015. Preliminary annotated list of the Lepidoptera of Sivas Province (East Turkey). Cesa News, 113: 1-101.

Koçak AÖ, Seven S 1996. Anadolunun Diurnal Lepidoptera Birlikleri ve Ekolojisi. Priamus, 8(3/4): 53-162.

Koçak AÖ, Kemal M 2009. Revised Checklist of the Lepidoptera of Turkey. Priamus (Suppl.), 17: 1-253.

Koçak AÖ 2014. List of the 23773 Pterygot species in Turkey based upon the info-system of the Cesa. Priamus (Supplement) 32: 1-876.

Lattin Gde 1950. Türkische Lepidopteren -I. Ist. Univ. Fen Fak. Mec. (Ser B) 15(4): 301-328.

Lattin Gde 1951. Türkische Lepidopteren -II. Ist. Univ. Fen Fak. Mec. (Ser B) 16(1): 45-73.

Leraut P 2014. Moths of Europe, Pralids 2. Vol 4. N.A.P. Editions, Verrières-le-Buisson, France. 69 pls, 190 text figs., $441 \mathrm{pp}$.

Okyar Z, Aktaç N 1999. Faunistic and taxonomic studies on the Geometridae species of Turkish Thrace. Turkish Journal of Zoology, 23, sup. 1, 99 132.

Özdemir M 2016. Contributions to the knowledge of Geometridae fauna (Lepidoptera) of Ordu province. Bitki Koruma Bülteni, 56(2): 209-225.
Parenti U, 2000. A Guide to the Microlepidoptera of Europe. Museo Regionale di Scienze Naturali, Torino, $426 \mathrm{pp}$.

Razowski J $1970 . \quad$ Cochylidae, [In] Amsel,H.G.,Reisser,H. \& \& F.Gregor: Microlepidoptera Palaearctica vol. 3 (Text \& Tafeln). Georg Fromme, Wien. 161 Taf., xiv + 528 pp.

Robinson, G. S., 1976. The Preparation of slides of Lepidoptera genitalia with special reference to the Microlepidoptera. Entomologist's Gazette, 27: 127132.

Roesler RU 1973. Trifine Acrobasiina (1. Teilband der Phycitinae). [in] Amsel, H.G. et al., Microlepidoptera Palaearctica vol.4 (Text \& Tafeln). Verlag G. Fromme Co. Wien, 159 Taf., 752 pp.

Roesler RU 1993. Quadrifine Acrobasiina Erster Teil (2. Teilband der Phycitinae). [in] Amsel, H.G., Microlepidoptera Palaearctica vol.8 (Text \& Tafeln). Verlag G.Braun, Karlsruhe, 82 Taf., 305 pp.

Sattler K 1967. Ethmiidae, [In] Amsel, H. G., Reisser, H. \& F. Gregor: Microlepidoptera Palaearctica vol. 2, Georg Fromme, Wien. 161 Taf., xiv + 528.

Seven E 2014. Siirt'in Şirvan ilçesinin Macroheterocera türleri üzerine eko-faunistik araştırmalar (Lepidoptera). YYÜ. Fen Bil. Enst., Biyoloji ABD, Doktora Tezi. [Yayınlanmamış]. xvii+ $428 \mathrm{~s}$.

Seven S 1996. Türkiye Lepidoptera faunası ve ekolojisi Üzerine araştırmalar-I. Cent. ent. Stud., Priamus 8 (1/2): $1-52$.

Slamka F 2006 (2011). Pyraloidea of Europe (Lepidoptera) Volume 1. Pyralinae, Galleriinae, Epipaschiinae, Cathariinae \& Odontiinae. Identification, Distribution, Habitat, Biologie. Bratislava, 1-139.

Slamka F 2008. Pyraloidea of Europe (Lepidoptera) Volume 2. Crambinae \& Schoenobiinae. Identification, Distribution, Habitat, Biologie. Bratislava, 223 pp.

Slamka F 2013. Pyraloidea of Europe (Lepidoptera) Volume 3. Pyraustinae \& Spilomelinae. Identification, Distribution, Habitat, Biologie. Bratislava, $357 \mathrm{pp}$.

Zukowsky B 1937. Reisebericht über entomologische Aufsammlungen im nordöstlichen Anatolien (westliches Armenien) 1934 (Lep.). Ent. Rdsch., 55: 1-6, 13-14, 27-30, 37-40.

Zukowsky B 1938. Herbstreise nach Kleinasien, Nordost-Anatolien und zilizischer Taurus (Lep.). Ent. Rdsch., 55: 529-531, 623-627, 648-651, 657-659, 708.

Zukowsky B 1941. Siwas und Akschehir 1937 (Lep.). Ent. Z., Frankf. a. M. 54: 266-272. 